\title{
Structural and metal-halogen exchange reactivity studies of sodium magnesiate biphenolate complexes
}

Received 00th January 20xx, Accepted 00th January 20xx DOI: $10.1039 / x 0 \times x 00000 x$
Callum Structural and metal-halogen exchange reactivity studies of sodium magnesiate biphenolate

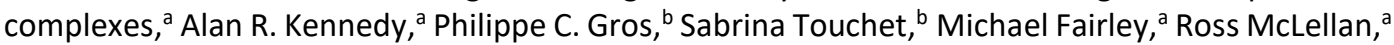
Antonio J. Martínez-Martínez ${ }^{\mathrm{c}}$ and Charles T. O’Hara*a

\begin{abstract}
Bimetallic sodium magnesiates have been employed in metal-halogen exchange for the first time. Utilising the racemic

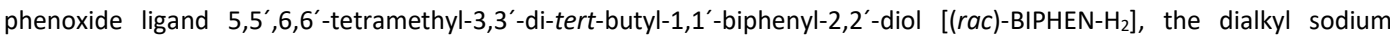
magnesiates [(rac)-BIPHEN]Na $\mathrm{MgBu}_{2}(\mathrm{TMEDA})_{2} 3$ and [(rac)-BIPHEN] $\mathrm{Na}_{2} \mathrm{MgBu}_{2}(\mathrm{PMDETA})_{2} 4$ have been synthesised. Both 3 and 4 can be easily prepared through co-complexation of di- $n$-butylmagnesium with the sodiated (rac)-BIPHEN precursor which can be prepared in situ in hydrocarbon solvent. Prior to the main investigation, synthesis of the sodiated precursor $[\mathrm{BIPHEN}]_{2} \mathrm{Na}_{4}(\mathrm{THF})_{4} 1$ was explored in order to better understand the formation of sodium magnesiates utilising the dianionic ( $r a c)$-BIPHEN ligand as the parent ligand. In addition, a BIPHEN-rich sodium magnesiate [BIPHEN] ${ }_{2} \mathrm{Na}_{2} \mathrm{Mg}(\mathrm{THF})_{4} 2$ was prepared and characterised, and its formation was rationalised. Complex $\mathbf{1}$ and $\mathbf{4}$ have also been fully characterised in both solid and solution state. In terms of onward reactivity, $\mathbf{3}$ and $\mathbf{4}$ have been tested as potential exchange reagents with aryl and heteroaryl iodides to produce aryl and heteroaryl magnesium phenoxides utilising toluene as a non-polar hydrocarbon solvent. Complex 3 reacted smoothly to give a range of aryl and heteroaryl magnesium phenoxides, whilst $\mathbf{4}$ 's reactivity is more sluggish.
\end{abstract}

\section{Introduction}

Metal-halogen exchange involving aromatic halides is a powerful transformation in synthetic chemistry. It allows the facile conversion of aromatic halides to useful functionalised arenes via metal aryl intermediates. Traditionally this exchange has been carried out utilising monometallic lithium- and magnesium-based reagents. Organolithium reagents typically require the use of cryogenic conditions and exhibit a poor functional group tolerance, whilst organomagnesiums often display a greater functional group tolerance but are generally less reactive. However, the reactivity of organomagnesium reagents can be increased by combining them with an alkal metal one to generate an alkali metal magnesiate. ${ }^{1}$ As structure is inextricably linked to reactivity in organomagnesium chemistry, and much like monometallic Grignard reagents whose solution-state structure is dictated by the Schlenk equilibrium, alkali metal magnesiates also show a structural complexity which might not be immediately evident or

a. WestCHEM, University of Strathclyde, 295 Cathedral Street, Glasgow, G1 1XL, UK. b. Université de Lorraine, CNRS, L2CM, F-54000 Nancy, France.

c. Supramolecular Organometallic Chemistry Laboratory CIOSO-Center for Research in Sustainable Chemistry \& Department of Chemistry University of Huelva Campus El Camen, 21007 Huelva, Spain

+ Dedicated to Professor Robert E. Mulvey, a fantastic mentor and colleague, on the occasion of his $60^{\text {th }}$ birthday.

Electronic Supplementary Information (ESI) available: [details of any supplementary information available should be included here]. See DOI: 10.1039/x0xx00000x expected. ${ }^{2,3}$ The synergic behaviour arising from the combination of two metal centres within a complex gives these reagents unique and often enhanced reactivity, allowing them to perform transformations that would otherwise be unachievable using their monometallic parent complexes. ${ }^{1,4,5}$ Not only do these bimetallic reagents allow for reactions to be carried out under milder conditions ${ }^{6}$ (i.e., carried out at temperatures at or close to ambient temperature), they also exhibit a much greater functional group tolerance compared to monometallic bases. ${ }^{7}$ Consequently, bimetallic magnesiates have attracted much attention for use in metal-halogen exchange reactions. Trialkylmagnesiates are also used extensively. ${ }^{8}$ The enhanced reactivity of these species can, in some cases, enable exchange to occur using sub-stoichiometric quantities of magnesiate. ${ }^{9}$ Despite their reactivity, it has been shown that exchange does not always occur with full consumption of all the alkyl ligands leading to undesired side reactions. ${ }^{10}$

Replacement of unreactive alkyl ligands with alkoxides is therefore an attractive principle for tuning the chemoselectivity of magnesiate complexes. Knochel and co-workers have recently utilised 2-ethylhexanol to synthesise alkylmagnesium alkoxides ${ }^{\mathrm{S} B u M g O R} \cdot \mathrm{LiOR}$ and ${ }^{\mathrm{S}} \mathrm{Bu}_{2} \mathrm{Mg} \cdot 2 \mathrm{LiOR}$ (where $\mathrm{R}=2$ ethylhexyl). ${ }^{11}$ These reagents undergo magnesium-halogen exchange with a range of aryl and hetero-aryl substrates at ambient temperatures in non-polar solvents. Furthermore, the inclusion of alkoxide ligands also provides facile access to the realm of asymmetric synthesis. ${ }^{12-16}$ Noyori has reported the 
efficient enantioselective alkylation of aldehydes using a lithium magnesiate containing the chiral BINOL alkoxide ligand. ${ }^{17}$ Furthermore, the groups of Gros and Mongin have utilised lithium magnesiates containing the chiral diols $(R, R)$-TADDOL and $(R)$-BIPHEN $[(R, R)$-TADDOL is $(4 R, 5 R)$-2,2-dimethyl-1,3dioxolane-4,5-diyl)bis(bis(4-(tert-butyl)phenyl)methanol and (R)-BIPHEN is $5,5^{\prime}, 6,6^{\prime}$-tetramethyl-3,3' $3^{\prime}$-di-tert-butyl-1, $1^{\prime}$ biphenyl-2,2'-diol] as alkoxide ligands in metal-halogen exchange reactions to access a range of chiral alcohols. ${ }^{18,19} \mathrm{~A}$ well-defined and robust organometallic complex with complete saturation of the metal coordination sites is essential in order to limit excess aggregation and subsequent loss of enantioselectivity. Our group has successfully characterised a series of dilithium dialkylmagnesiates containing the (rac)BIPHEN ligand and demonstrated their reactivity in metalhalogen exchange. ${ }^{20}$ Using the enantiomerically pure form of the ligand, (S)-BIPHEN, Gros and co-workers have synthesised a range of chiral 3-substituted isobenzofuranones with good enantiomeric ratios and yields from reaction of the monoalkyl lithium magnesiate (S)-BIPHENnBuMgLi with ethyl-2iodobenzoate and subsequent electrophilic quenching with a range of aldehydes. ${ }^{21}$

Despite the wealth of knowledge relating to the structure of lithium magnesiates and their reactivity in metal-halogen exchange, the same cannot be said for sodium magnesiates, particularly those bearing alkoxide ligands. Sodium magnesiates have primarily been employed as powerful bases in deprotonation chemistry ${ }^{22,23}$ and as efficient catalysts for a range of transformations. ${ }^{24-28}$ To the best of our knowledge there are no examples of metal-halogen exchange involving sodium magnesiates. Herein, we report the synthesis of two new disodium dialkylmagnesiates containing the (rac)-BIPHEN ligand (Scheme 1) and discuss their preliminary reactivity in metal-halogen exchange.

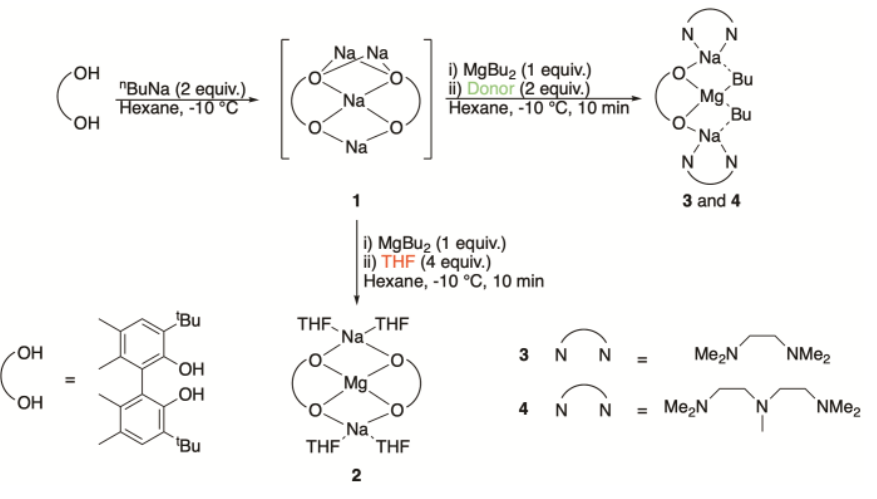

Scheme 1: Preparation of sodium dialkylmagnesiates.

\section{Results and Discussion}

\section{Syntheses of Sodium Magnesiates from (rac)-BIPHEN}

Prior to preparing the desired sodium magnesiates, synthesis of the homometallic sodium precursor was initiated starting from the ( $r a c)$-BIPHEN ligand. The parent ( $r a c)-B I P H E N-\mathrm{H}_{2}$ ligand was doubly deprotonated using nuNa (two molar equivalents) in hexane solution. To aid crystallisation, the majority of the hexane was removed in vacuo and replaced with THF. Cooling the resultant solution to $-18{ }^{\circ} \mathrm{C}$ generated the desired disodium

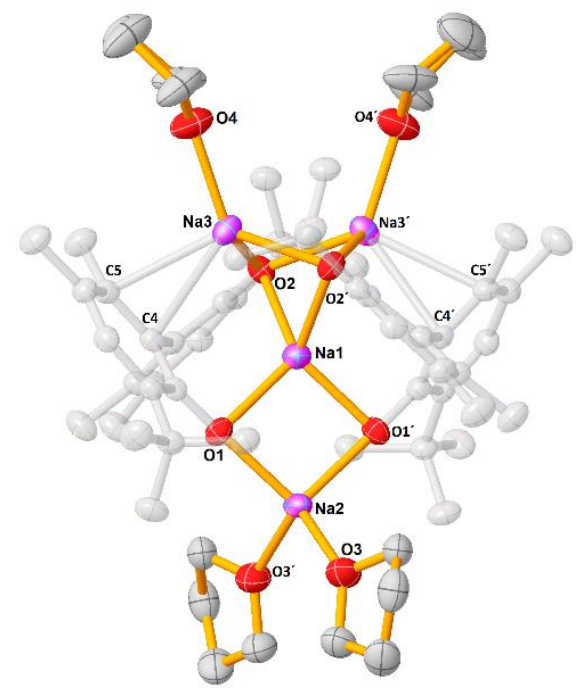

phenoxide

Figure 1: Solid state structure of $[\mathrm{BIPHEN}]_{2} \mathrm{Na}_{4}(\mathrm{THF})_{4} \cdot 0.4(\mathrm{THF})$ 1. Hydrogen atoms and disorder in THF molecules are omitted for clarity. Thermal ellipsoids are set to $50 \%$ probability level. Key bond distances $(\AA ̊)$ and bond angles $\left({ }^{\circ}\right): \mathrm{Na}(1)-\mathrm{O}(1)$, 2.217(2); $\mathrm{Na}(1)-\mathrm{O}\left(1^{\prime}\right), 2.217(2) ; \mathrm{Na}(1)-\mathrm{O}(2), 2.313(2) ; \mathrm{Na}(1)-\mathrm{O}\left(2^{\prime}\right), 2.313(2) ; \mathrm{Na}(2)$ $\mathrm{O}(1), 2.199(2) ; \mathrm{Na}(2)-\mathrm{O}\left(1^{\prime}\right), 2.199(2) ; \mathrm{Na}(2)-\mathrm{O}(3), 2.308(3) ; \mathrm{Na}(2)-\mathrm{O}\left(3^{\prime}\right), 2.308(3)$; $\mathrm{Na}(3)-\mathrm{O}(2), 2.265(2) ; \mathrm{Na}(3)-\mathrm{O}\left(2^{\prime}\right), 2.298(2) ; \mathrm{Na}(3)-\mathrm{O}(4), 2.298(3) ; \mathrm{Na}(3)-\mathrm{C}(4)$, 2.878(3); $\mathrm{Na}(3)-\mathrm{C}(5), 2.808(3) ; \mathrm{Na}\left(3^{\prime}\right)-\mathrm{O}(2), 2.298(2) ; \mathrm{Na}\left(3^{\prime}\right)-\mathrm{O}\left(2^{\prime}\right), 2.265(2) ;$ $\mathrm{Na}\left(3^{\prime}\right)-\mathrm{O}\left(4^{\prime}\right), 2.298(3) ; \mathrm{O}(1)-\mathrm{Na}(1)-\mathrm{O}\left(1^{\prime}\right), 92.49(11) ; \mathrm{O}(1)-\mathrm{Na}(1)-\mathrm{O}(2), 110.87(7) ;$ $\mathrm{O}(2)-\mathrm{Na}(1)-\mathrm{O}\left(2^{\prime}\right), \quad 78.01(10) ; \quad \mathrm{O}(1)-\mathrm{Na}(2)-\mathrm{O}\left(1^{\prime}\right), 93.45(12), \quad \mathrm{O}(3)-\mathrm{Na}(2)-\mathrm{O}\left(3^{\prime}\right)$, 103.62(14); $\mathrm{O}(2)-\mathrm{Na}(3)-\mathrm{O}\left(2^{\prime}\right), 79.27(8) ; \mathrm{O}(2)-\mathrm{Na}\left(3^{\prime}\right)-\mathrm{O}\left(2^{\prime}\right), 79.27(8)$.

[BIPHEN] ${ }_{2} \mathrm{Na}_{4}(\mathrm{THF})_{4} 1$ (unoptimised $26 \%$ crystalline yield). In an attempt to prepare the desired magnesiate, a hexane solution of the sodium reagent was generated in-situ as described herein, followed by co-complexation with ${ }^{\mathrm{n}} \mathrm{Bu}_{2} \mathrm{Mg}$ (one molar equivalent). THF was added and the mixture heated gently to ensure complete dissolution. After cooling to $-18^{\circ} \mathrm{C}, \mathrm{X}$-ray quality colourless crystals were isolated and were determined to be the nBu-free [BIPHEN rich] complex [BIPHEN] ${ }_{2} \mathrm{MgNa}_{2}(\mathrm{THF})_{4} \mathbf{2}$ (37\% crystalline yield. Maximum yield of $50 \%$ based on metals). In an attempt to prepare heteroleptic (mixed alkyl/phenoxide) magnesiates, $N, N, N^{\prime}, N^{\prime}$ tetramethylethylenediamine (TMEDA) and $N, N, N^{\prime}, N^{\prime \prime}, N^{\prime \prime}$ pentamethylethylenetriamine (PMDETA) were utilised as chelating donor ligands. To emphasise, instead of using THF in the aforementioned reaction, TMEDA and PMDETA (two molar equivalents) were utilised. Using this slightly modified synthetic methodology, the higher order magnesiates [(rac)BIPHEN]Na $\mathrm{Mgn}^{\mathrm{n}} \mathrm{Bu}_{2}(\mathrm{TMEDA})_{2} \quad 3 \quad(56 \%$ yield $)$ and $[(\mathrm{rac})-$ BIPHEN]Na ${ }_{2} \mathrm{Mgn}^{\mathrm{n}} \mathrm{Bu}_{2}(\mathrm{PMDETA})_{2} 4$ (55\% yield) were synthesised.

\section{X-ray crystallographic studies}

The structure of the disodium phenoxide, which crystallised as a dimeric THF solvate, was revealed to be the tetranuclear complex [BIPHEN $]_{2} \mathrm{Na}_{4}(\mathrm{THF})_{4}$ 1. Each molecule has crystallographically imposed 2-fold symmetry, and as two of the $\mathrm{Na}$ centres sit on the twofold axis, there are three chemically distinct sodium environments (Figure 1). Despite using the 
racemic form of the BIPHEN ligand during synthesis, the crystal of 1 measured was enantiopure, chiral space group $P 4_{1} 2_{1} 2$, with one crystallographically unique BIPHEN ligand per asymmetric unit [Flack parameter, $0.018(14)]$. Spontaneous resolution upon crystallisation is thought to occur for approximately 5 to $10 \%$ of all racemic compounds. This forms conglomerates, or physical mixtures, of separate $R$ - and $S$-crystals. ${ }^{29,30}$ In the absence of any driver to enantiopurity, this is presumably the case here.

Analysis of the organometallic framework of $\mathbf{1}$ shows the central sodium atom $\mathrm{Na} 1$ is four coordinate with a distorted tetrahedral geometry (sum of bond angles $664.1^{\circ}$ ) and is bound to all four oxygen atoms of both BIPHEN ligands [range of $\mathrm{Na}-\mathrm{O}$

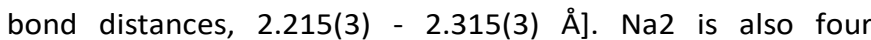
coordinate with a distorted tetrahedral geometry (sum of bond angles, $\left.661.1^{\circ}\right)$ and is bound to one oxygen atom from each BIPHEN ligand [Na2-O1 2.200(3) Å]. The coordination sphere of $\mathrm{Na} 2$ is complete by coordination to two molecules of THF [ Na2O3 2.310(4) Å]. The other sodium sites $\mathrm{Na3} / \mathrm{Na3}^{\prime}$ each make short contacts with 5 atoms. Each such Na centre coordinates to three $\mathrm{O}$ atoms [one from each BIPHEN ligand and one from a THF molecule]. The remaining contacts are $\eta^{2}-\pi$-interactions to an adjacent phenyl group [ $\mathrm{Na3}-\mathrm{C} 4$ and $\mathrm{Na3}-\mathrm{C} 5$ bond distances, $2.878(3)$ and $2.808(3) \AA$ respectively]. Comparison of the key bond lengths and bond angles of 1 with its related tetranuclear lithium complex (BIPHEN) ${ }_{2} \mathrm{Li}_{4}(\mathrm{THF})_{4}$ reported in our previous work, ${ }^{20}$ revealed an enhanced puckering of the four membered ring at the top of the structure ( $\mathrm{Na3}-\mathrm{O} 2-\mathrm{Na}^{\prime}-\mathrm{O}_{2}^{\prime}$ ring in Figure 1). For a comparison see the Supporting Information.

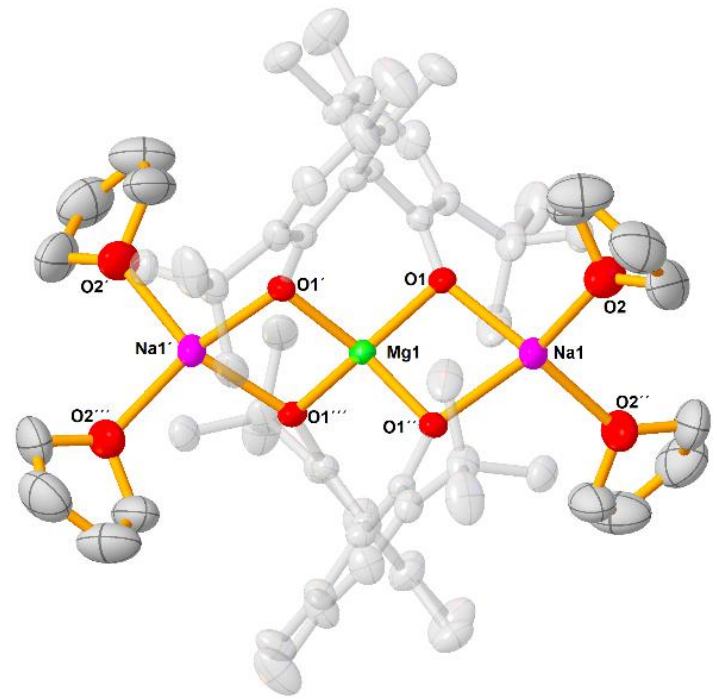

Figure 2: Solid-state structure of $[\mathrm{BIPHEN}]_{2} \mathrm{MgNa}_{2}(\mathrm{THF})_{4}$ 2. Hydrogen atoms and disorder in THF molecules are omitted for clarity. Thermal ellipsoids are set to 50 $\%$ probability level. Key bond distances $(\AA)$ and bond angles $\left({ }^{\circ}\right)$ : $\mathrm{Mg}(1)-\mathrm{O}(1)$ 1.960(16); $\mathrm{Mg}(1)-\mathrm{Na}(1), \quad 3.0914(14) ; \mathrm{Mg}(1)-\mathrm{Na}\left(1^{\prime}\right), 3.0913(14) ; \mathrm{Na}(1)-\mathrm{O}(1)$ 2.2372(19); $\mathrm{Na}(1)-\mathrm{O}(2), 2.270(7) ; \mathrm{O}(1)-\mathrm{Mg}(1)-\mathrm{O}\left(1^{\prime}\right), 102.72(9) ; \mathrm{O}(1)-\mathrm{Mg}(1)-\mathrm{O}\left(1^{\prime \prime}\right)$ 92.32(9); $\mathrm{O}(1)-\mathrm{Mg}(1)-\mathrm{O}\left(1^{\prime \prime \prime}\right), 137.67(10) ; \mathrm{Na}(1)-\mathrm{Mg}(1)-\mathrm{Na}\left(1^{\prime}\right), 180 ; \mathrm{O}(1)-\mathrm{Na}(1)$ $\mathrm{O}\left(1^{\prime \prime}\right), 78.38(38) ; \mathrm{O}(2)-\mathrm{Na}(1)-\mathrm{O}\left(2^{\prime \prime}\right), 101.5(2)$

Turning to the BIPHEN-rich $\mathbf{2}$ which also crystallised with crystallographically imposed symmetry. The central magnesium centre Mg1 sits on three twofold axes and is four coordinate with a distorted tetrahedral geometry (sum of bond angles $665.6^{\circ}$ ). The Mg-O bond length is $1.962(3) \AA$ and this is in good agreement with its homometallic analogue. ${ }^{31}$ The related sodium atom sites $\mathrm{Na} 1 / \mathrm{Na}^{\prime}{ }^{\prime}$ are situated on a twofold axis and are also four coordinate, again adopting a distorted tetrahedral geometry (sum of bond angles $662.1^{\circ}$ ). They are bound to a single oxygen atom of each of the BIPHEN ligands [Na1-O1 bond distance, 2.229(4) $\AA$ ] and their coordination spheres are completed by two molecules of THF [Na1-O2 bond distance, 2.273(5) Å]. Similar to the situation observed with 1, the single crystal of $\mathbf{2}$ examined was determined to be chiral, crystallising in the chiral space group 1222 [Flack parameter, -0.16(17)].

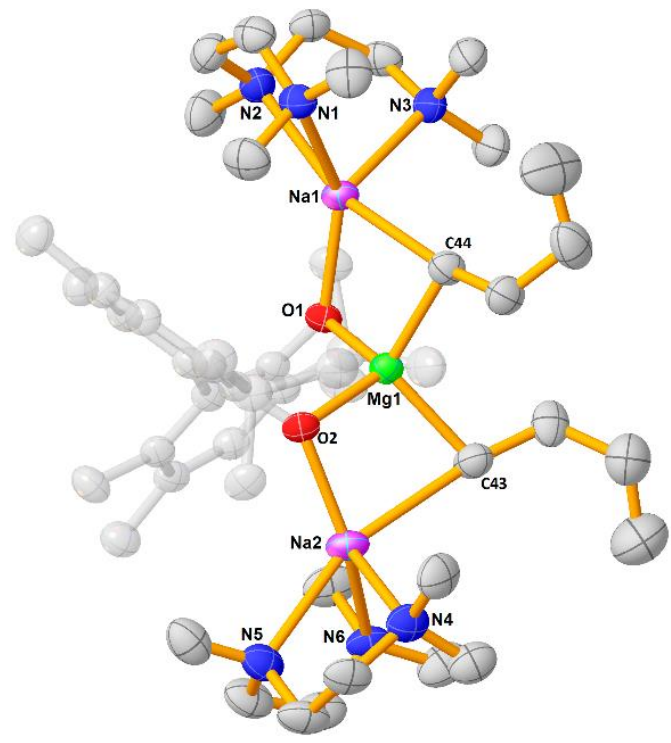

Figure 3: Solid state structure of [(rac)-BIPHEN] $\mathrm{Na}_{2} \mathrm{MgBu}_{2}(\mathrm{PMDETA})_{2} 4$. Hydrogen atoms and disorder in PMDETA molecules are omitted for clarity. Thermal ellipsoids are set to $50 \%$ probability level. Key bond distances $(\AA)$ and bond angles $\left({ }^{\circ}\right): \mathrm{Mg}(1)-\mathrm{O}(1), 2.011(3) ; \mathrm{Mg}(1)-\mathrm{O}(2), 2.009(3) ; \mathrm{Mg}(1)-\mathrm{C}(43), 2.203(6) ; \mathrm{Mg}(1)-$ $\mathrm{C}(44), 2.204(5) ; \mathrm{Na}(1)-\mathrm{O}(1), 2.279(3) ; \mathrm{Na}(1)-\mathrm{N}(1), 2.614(4) ; \mathrm{Na}(1)-\mathrm{N}(2), 2.558(4) ;$ $\mathrm{Na}(1)-\mathrm{N}(3), 2.520(4) ; \mathrm{Na}(2)-\mathrm{O}(2), 2.267(3) ; \mathrm{Na}(2)-\mathrm{N}(4), 2.492(5) ; \mathrm{Na}(2)-\mathrm{N}(6)$, 2.618(4); $\mathrm{Na}(1)-\mathrm{C}(44), 2.690(5) ; \mathrm{Na}(2)-\mathrm{C}(43), 2.688(6) ; \mathrm{O}(1)-\mathrm{Mg}(1)-\mathrm{O}(2), 99.35(13)$, $\mathrm{C}(43)-\mathrm{Mg}(1)-\mathrm{C}(44), \quad$ 107.7(2); $\mathrm{Na}(1)-\mathrm{C}(44)-\mathrm{Mg}(1), \quad 79.3(2) ; \quad \mathrm{Na}(2)-\mathrm{C}(43)-\mathrm{Mg}(1)$ 79.1(2); O(1)-Na(1)-C(44), 82.0(1); O(2)- $\mathrm{Na}(2)-\mathrm{C}(43), 91.9(3)$.

Introduction of the tridentate donor PMDETA lead to isolation of [(rac)-BIPHEN]Na $\mathrm{MgBu}_{2}(\mathrm{PMDETA})_{2}$. Interestingly, complex 4 crystallised in the $P_{n}$ space group indicating the racemic form of the BIPHEN ligand is retained. In keeping with the other magnesiates prepared in this work, the central magnesium atom of $\mathbf{4}$ was found to be four coordinate with a distorted tetrahedral geometry (sum of bond angles $658.6^{\circ}$ ). It is bound to the (rac)-BIPHEN ligand in a chelating fashion and to two butyl ligands. The $\mathrm{Mg}-\mathrm{O}$ bond lengths were found to be identical within experimental error [Mg1-O1 2.011(3) $\AA$ and Mg1-O2 2.009(3) $\AA$ ] as were the $\mathrm{Mg}-\mathrm{C}$ bond lengths [Mg1-C42 2.203(6) $\AA$ and Mg1-C44 2.204(3) $\AA$ ] making the bonding symmetrical in each case. Sodium atoms $\mathrm{Na} 1$ and $\mathrm{Na} 2$ are five coordinate, each forming a bond to the oxygen atoms of the ( $\mathrm{rac}$ )-BIPHEN ligand (Na1-O1 2.279(3) $\AA$ and Na2-O2 2.267(3) $\AA$ ) and an interaction with the terminal methylene group, the $\alpha$-carbon, of the butyl chains. The sodium to alkyl carbon bond distances were

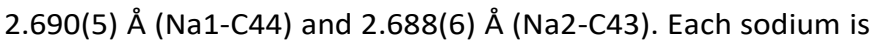


capped by a tridentate PMDETA ligand [Na1-N1, 2.614(4) $\AA$;

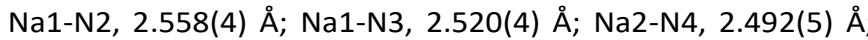

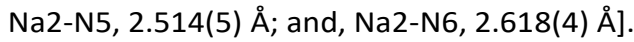

\section{Solution studies}

The isolation of the BIPHEN-rich complex $\mathbf{2}$ is perhaps surprising considering the stoichiometry of starting reagents used in the reaction. It can be attributed to the disproportionation (ligand reorganisation) of the expected disodium dialkyl magnesiate product, [(rac)-BIPHEN]MgNa $\mathrm{Bu}_{2}(\mathrm{THF})_{4}$ (Scheme 2). This type of reorganisation is a common pathway to generally undesired compounds in related bimetallic chemistry. ${ }^{32,33}$ It is possible that the formation of $\mathbf{2}$ may be due to thermal decomposition however, no other products from this Schlenk-like equilibrium

\begin{tabular}{|c|c|c|}
\hline & THF & {$[(r a c)-B I P H E N]_{2} \mathrm{Na}_{2} \mathrm{Mg}(\mathrm{THF})_{4}$} \\
\hline $2[(r a c)-B I P H E N] N_{2} \mathrm{MgBu}_{2}(\mathrm{THF})_{4}$ & & $\begin{array}{c}\stackrel{+}{+} \\
\mathrm{Na}_{2} \mathrm{MgBu}_{4}(\mathrm{THF})_{4}\end{array}$ \\
\hline
\end{tabular}

Scheme 2: Simplified Schlenk equilibrium demonstrating the formation of 2.

were isolated from the reaction (Scheme 2). The reaction was subsequently repeated using the bulkier dialkylmagnesium reagent $\mathrm{Mg}\left(\mathrm{CH}_{2} \mathrm{SiMe}_{3}\right)_{2}$. Co-complexation of $\mathrm{Mg}\left(\mathrm{CH}_{2} \mathrm{SiMe}_{3}\right)_{2}$ with 1 again produced the BIPHEN-rich 2 as the only isolable reaction product and not the desired magnesiate [(rac)BIPHEN]Na $\mathrm{MgR}_{2}(\mathrm{THF})_{4}$. Despite multiple attempts at preparing both dialkylmagnesiates, their solid-state structure could not be determined - the only isolable product on each occasion was $\mathbf{2}$.

In an attempt to preclude the aforementioned Schlenk-like equilibrium, the bidentate donor TMEDA was employed. It was expected that the strong chelation of the diamine with the $\mathrm{Na}$ centre may inhibit further reaction to form undesired $\mathbf{2}$. This was indeed the case and the formation and isolation of [(rac)BIPHEN]Na $\mathrm{Mg}^{\mathrm{n}} \mathrm{Bu}_{2}$ (TMEDA) ${ }_{2} \mathbf{3}$ was facile. As noted previously, by employing PMDETA, the related complex 4 was prepared. The excellent solubilities of $\mathbf{3}$ and $\mathbf{4}$ in arene solvents has allowed them to be characterised by ${ }^{1} \mathrm{H}$ and ${ }^{13} \mathrm{C} N M R$ spectroscopy (Tables 2 and 3). The $\mathrm{CH}_{2}-\mathrm{Mg}$ methylene hydrogen atoms for 3 appear at $\delta-0.31$ and -0.78 ppm as two broad multiplets rather than a single resonance due to the diastereotopic nature of these hydrogen atoms. The corresponding $\mathrm{CH}_{2}-\mathrm{Mg}$ was located at $8.84 \mathrm{ppm}$ in the ${ }^{13} \mathrm{C} \mathrm{NMR}$ spectrum. Comparison of the ${ }^{1} \mathrm{H}$ and ${ }^{13} \mathrm{C}$ NMR spectra of 3 with those of $\mathbf{4}$ showed a good level of agreement with the exception of the resonance

Table 1: Key ${ }^{1} \mathrm{H}$ NMR chemical shifts for $\mathbf{3}$ and $\mathbf{4}$ in $\mathrm{C}_{6} \mathrm{D}_{6}$ at $300 \mathrm{~K}$

\begin{tabular}{c|c|c} 
& \multicolumn{2}{|c}{${ }^{1}$ H Chemical Shift $(p p m)$} \\
& $\mathbf{3}$ & $\mathbf{4}$ \\
\hline $\mathrm{Ar}$ & 7.18 & 7.14 \\
$\mathrm{t} \mathrm{Bu}$ & 1.70 & 1.77 \\
$\mathrm{Me}$ & $1.80,2.23$ & $1.90,2.31$ \\
Mg-CH & $-0.78,-0.31$ & -0.75 \\
TMEDA & $1.79,1.86$ & - \\
PMDETA & - & $1.82,1.86,1.93$
\end{tabular}

associated with the $\mathrm{Mg}-\mathrm{CH}_{2} \mathrm{H}$ atoms which appear as one broad multiplet at $-0.75 \mathrm{ppm}$, instead of two distinct multiplets. A low temperature NMR spectroscopic study failed to separate this multiplet (see Supporting Information). The data for $\mathbf{3}$ and $\mathbf{4}$ are consistent with values reported in literature for the related lithium magnesiates. ${ }^{20}$

To further investigate the role in which TMEDA and PMDETA play in suppressing disproportionation, $C_{6} \mathrm{D}_{6}$ and $\mathrm{d}_{8}-\mathrm{THF}$ solutions of complexes $\mathbf{3}$ and $\mathbf{4}$ were prepared. Immediate displacement of TMEDA and PMDETA by THF was observed for both 3 and 4 in $d_{8}$-THF. Monitoring the solutions by ${ }^{1} H$ NMR spectroscopy showed no disproportion occurred in either solution of $\mathbf{3}$ or $\mathbf{4}$ at $300 \mathrm{~K}$. Heating these solutions to $50^{\circ} \mathrm{C}$ for a further 12 hours showed no change in ${ }^{1} \mathrm{H}$ NMR spectra of $\mathbf{3}$ or $\mathbf{4}$ in $\mathrm{C}_{6} \mathrm{D}_{6}$. In $\mathrm{d}_{8}$-THF however, complex 3 showed multiple new resonances in the aliphatic and aromatic regions of the ${ }^{1} \mathrm{H} N M R$ spectrum which was accompanied by a colour change in the solution from colourless to yellow. Surprisingly, the ${ }^{1} \mathrm{H} N M R$ spectrum of 4 in $\mathrm{d}_{8}$-THF showed comparatively little decomposition compared to that of complex $\mathbf{3}$ under the same conditions and suggests the parent magnesiate 4 remains intact i.e., no ligand rearrangement occurs after heating in $\mathrm{d}_{8}$-THF (see Supporting Information).

Table 2: Key ${ }^{13} \mathrm{C}$ NMR chemical shifts for $\mathbf{3}$ and $\mathbf{4}$ in $\mathrm{C}_{6} \mathrm{D}_{6}$ at 300K.

\begin{tabular}{c|c|c} 
& \multicolumn{2}{|c}{${ }^{13}$ C Chemical Shift $(\mathrm{ppm})$} \\
& $\mathbf{3}$ & $\mathbf{4}$ \\
\hline $\mathrm{Ar}$ & $119.6,127.2$, & $119.8,126.5$, \\
& $132.1,132.7$, & $132.6,134.1$, \\
& $135.9,162.7$ & $134.8,163.0$ \\
tBu & $31.7,35.3$ & $32.2,35.4$ \\
Me & $17.4,20.7$ & $17.9,20.8$ \\
Mg-CH & 8.8 & 8.7 \\
TMEDA & $45.5,56.9$ & - \\
PMDETA & - & $44.1,45.5,54.0$
\end{tabular}

\section{Metal-halogen exchange studies}

The reactivity of $\mathbf{3}$ and $\mathbf{4}$ were investigated to assess their potential in metal-halogen exchange reactions ultimately generating of aryl and heteroaryl magnesium reagents (5-10, Table 3). Investigations into $\mathrm{Mg}-\mathrm{I}$ exchange were performed using a 2:1 ratio of organic substrate to exchange reagent (3 or 4) in order to achieve high efficiency double Mg-I exchange. Initially examining the reactivity of substrates bearing electron donating functionalities, the reaction of $\mathbf{3}$ and $\mathbf{4}$ with 2iodoanisole produced $\mathbf{5}$ in a 60 and 30\% yield respectively after 15 minutes. Concomitant formation of 4-iodobutane was observed as evidenced by resonances at $\delta 2.76,1.43,1.13$ and $0.69 \mathrm{ppm}$ in the associated ${ }^{1} \mathrm{H}$ NMR spectra in both cases. Formation of an additional product [ $29 \%$ yield for 3 and (41\%) for $4, \delta 3.33 \mathrm{ppm}$ ] was tentatively assigned to be the intermediate monoalkyl-monoaryl-magnesium species resulting from exchange of a single alkyl ligand. Continued monitoring of the reactions over a further 12 hours showed an increase in the yield of $\mathbf{5}$ to $90 \%$ with full consumption of $\mathbf{3}$, 
while a maximum yield of $77 \%$ was achievable using 4 . Furthermore, no changes were observed in the ${ }^{1} \mathrm{H}$ NMR spectra after full consumption of $\mathbf{3}$ and $\mathbf{4}$ which indicates no subsequent reaction between the newly formed diaryl magnesiate and the iodobutane generated in situ.

Table 3: Metal-halogen exchange of aryl iodides using $\mathbf{3}$ and $\mathbf{4}$.<smiles>Ic1c[R]ccc1</smiles>

$$
\underset{\mathrm{d}_{8} \text {-Toluene }}{\stackrel{[\mathrm{Mg}]-{ }^{\mathrm{n}} \mathrm{Bu}_{2}}{(0.5 \text { equiv. })}}
$$$$
25^{\circ} \mathrm{C}
$$

$[\mathrm{Mg}]=(\mathrm{rac})-\mathrm{BIPHENNa}{ }_{2} \mathrm{Mg}(\mathrm{TMEDA})_{2}$ for 3 $[\mathrm{Mg}]=(\mathrm{rac})-\mathrm{BIPHENNa}{ }_{2} \mathrm{Mg}(\mathrm{PMDETA})_{2}$ for 4

Product Substrate

Metal-Halogen Exchange Product
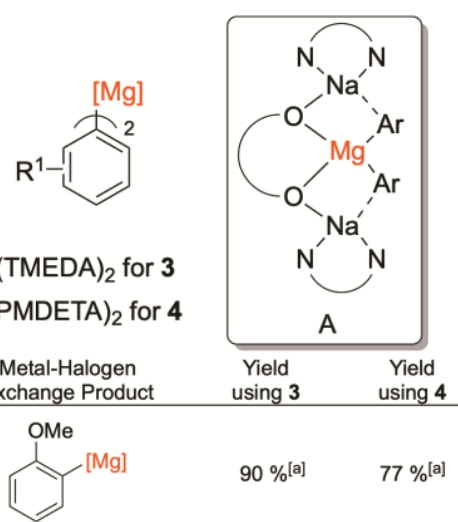
using using 4
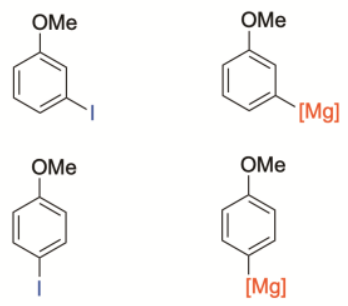

$90 \%$ [a]

$77 \%[$ [a]
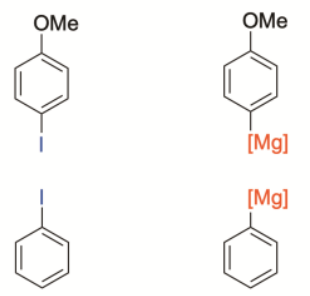

$79 \%[$ [b $20 \%[b]$

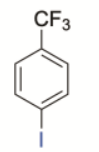

10
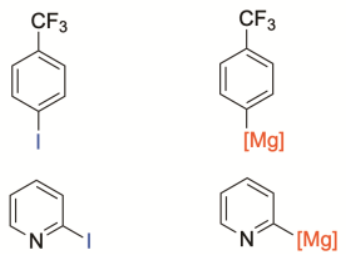

$87 \%[d]$

$84 \%[a]$

$78 \%[d]$

$80 \%[\mathrm{~d}]$

Reactions were performed in a Young's cap NMR tube using $0.0377 \mathrm{mmol}$ of magnesiate and $0.0754 \mathrm{mmol}$ of lodoarene. Unless otherwise stated yields were calculated from ${ }^{1} \mathrm{H}$ NMR spectroscopic data by integration against an internal standard of 1,2,3,4-tetraphentInapthalene. [a]Full consumption of magnesiate observed within 18 hours. ${ }^{[b]}$ Incomplete conversion of magnesiate after 18 hours. [c] Calculated from ${ }^{1} \mathrm{H}$ NMR spectroscopic data by integration against an internal standard of hexamethylcyclotrisiloxane. [d] Full consumption of magnesiate observed within 15 minutes.

Having demonstrated the ability of $\mathbf{3}$ and $\mathbf{4}$ to perform magnesium-halogen exchange reactions, the scope was expanded to investigate the tolerance of both magnesiates towards meta- and para-substituted electron rich substrates. Reaction with 3- and 4-iodoanisole produced 6 and 7 in considerably diminished yields of $14 \%$ and $6 \%$ respectively after 15 minutes using 3 while no appreciable reactivity was observed using 4. This significantly decreased reactivity is likely due to the more remote nature of the Lewis basic $\mathrm{MeO}$-group with respect to the site of metal-halogen exchange c.f., Directed orthoMetallation (DoM) chemistry. ${ }^{34}$ After 1 hour an increase in the yields of 6 and 7 to $41 \%$ and 20\% respectively were observed using 3 whilst with 4, 6 was obtained in only a 6\% yield and no formation of $\mathbf{7}$ was observed. In all cases there were significant quantities of the starting material 4-iodoanisole remaining. Continued monitoring of the reaction gave a maximum yield of 91\% for 6 accompanied by full consumption of $\mathbf{3}$ whilst a maximum conversion of $79 \%$ was observed for $\mathbf{7}$ without complete consumption of the parent magnesiate after an additional 12 hours. In the case of $\mathbf{4}$, full consumption of the magnesiate was not observed in either case and gave $\mathbf{6}$ and $\mathbf{7}$ in yields of $65 \%$ and $20 \%$ respectively. Complexes 3 and 4 were next tested with iodobenzene and furnished $\mathbf{8}$ in a low yield of $10 \%$ after 15 minutes using $\mathbf{3}$ whilst $\mathbf{4}$ again showed no reaction. After 1 hour, 8 was obtained in a $28 \%$ yield using 3 ; however, no increase in the yield was observed in the case of 4 . Full conversion of $\mathbf{3}$ to $\mathbf{8}$ was observed after prolonged monitoring to give a maximum yield of $90 \%$ whilst 4 remained only partially consumed giving 8 in a moderate yield of $57 \%$.

Turning to substrates containing electron withdrawing functionalities, the reactivity of $\mathbf{3}$ and $\mathbf{4}$ were investigated using 4-iodobenzotrifluoride. Complex 3 produced 9 in an $87 \%$ yield after less than 15 minutes with no observable increase in the yield after 1 hour. Complex 4 reacted slower producing 9 in yields of $24 \%$ and $56 \%$ after 15 minutes and 1 hour respectively. Conversion of 4 to 9 reached a maximum yield of $84 \%$ after a further 12 hours of reaction time. The tolerance of heteroaromatic substrates was next examined. Thus, complexes $\mathbf{3}$ and $\mathbf{4}$ were reacted with 2-iodopyridine to give $\mathbf{1 0}$ in yields of $78 \%$ and $80 \%$ respectively within 15 minutes. Despite the sluggish reactivity of $\mathbf{4}$ compared to $\mathbf{3}$ for the majority of substrates, reaction with the electron deficient 2iodopyridine gave a comparable yield. No increase in the yield of $\mathbf{5 f}$ was observed over time using $\mathbf{3}$ or $\mathbf{4}$. Perhaps counterintuitively, 3/4 appear to react slower than their THFsolvated lithium analogues in metal-halogen exchange reaction. ${ }^{20}$ This comparison has to be taken tentatively due to the difference in Lewis bases solvating the different metal centres. Intriguingly, a lower reactivity could potentially benefit selectivity/enantioselectivity which will be studied in due course.

\section{Conclusions}

Four novel organometallic complexes starting from the (rac)BIPHEN ligand have been characterised in both solid (1, 2 and 4) and solution-state (1-4). The heterobimetallic sodium magnesiates $\mathbf{3}$ and $\mathbf{4}$ been prepared and utilised in metalhalogen exchange reactions using a range of electron rich and electron poor substrates. Magnesiate $\mathbf{3}$ generally possesses high levels of reactivity in non-polar solvents particularly with electron deficient substrates; whereas, $\mathbf{4}$ is largely less reactive. It is possible that this difference in reactivity may be exploited in the future as it is likely that $\mathbf{4}$ will display higher levels of selectivity due to its lower reactivity. To the best of our knowledge these reactions represent the first examples of metal-halogen exchange utilising sodium magnesiates. Building on these results, the next stage in our research will utilise the enantiomerically pure ligands and apply the resulting magnesiates in asymmetric synthesis. 


\section{Conflicts of interest}

There are no conflicts to declare.

\section{Acknowledgements}

We gratefully acknowledge the Leverhulme Trust (research grant RPG-2016-281 to $\mathrm{CTOH)}$ and the Royal Society (International Exchange grant IEC \R2\170207 to CTOH and ST) for the generous sponsorship of this research. The data used within this publication can be accessed at xxxxx. The X-ray data have been deposited with the CCDC and are available from the data centre on quoting reference numbers 1988061-1988063.

\section{References}

1 S. D. Robertson and R. E. Mulvey, Top. Organomet. Chem., 2013, 45, 103-140.

2 A. J. Martínez-Martínez and C. T. O'Hara, Adv. Organomet. Chem., 2016, 65, 1-46.

J. Francos, B. J. Fleming, P. García-Álvarez, A. R. Kennedy, K. 2 Reilly, G. M. Robertson, S. D. Robertson and C. T. O'Hara, Dalton Trans., 2014, 43, 14424-14431. R. E. Mulvey, Organometallics, 2006, 25, 1060-1075. R. E. Mulvey, Acc. Chem. Res., 2009, 42, 743-755. S. Zaragoza-Calero, J. Francos, A. R. Kennedy and C. T. O'Hara, Dalton Trans., 2015, 44, 7258-7267. D. S. Ziegler, B. Wei and P. Knochel, Chem. Eur. J., 2019, 25 2695-2703. D. Tilly, F. Chevallier, F. Mongin and P. C. Gros, Chem. Rev., 2014, 114, 1207-1257. T. lida, T. Wada, K. Tomimoto and T. Mase, Tetrahedron Lett., 2001, 42, 4841-4844. A. Inoue, K. Kitagawa, H. Shinokubo and K. Oshima, J. Org. Chem., 2001, 66, 4333-4339. D. S. Ziegler, K. Karaghiosoff and P. Knochel, Angew. Chem. Int. Ed., 2018, 57, 6701-6704. H. G. Richey and J. P. DeStephano, J. Org. Chem., 1990, 55, 3281-3286. F. F. Fleming, V. Gudipati and O. W. Steward, Org. Lett., 2002, 4, 659-661. F. F. Fleming, S. Gudipati, V. A. Vu, R. J. Mycka and P. Knochel, Org. Lett., 2007, 9, 4507-4509. J. Farkas, S. J. Stoudt, E. M. Hanawalt, A. D. Pajerski and H. G. Richey, Organometallics, 2004, 23, 423-427. J. E. Chubb and H. G. Richey, Organometallics, 2002, 21 , 3661-3666. R. Noyori, S. Suga, K. Kawai, S. Okada and M. Kitamura, Pure Appl. Chem., 1988, 60, 1597-1606. O. Payen, F. Chevallier, F. Mongin and P. C. Gros, Tetrahedron Asymmetry, 2012, 23, 1678-1682. D. Catel, F. Chevallier, F. Mongin and P. C. Gros, Eur. J. Org. Chem., 2012, 53-57. J. Francos, P. C. Gros, A. R. Kennedy and C. T. O'Hara, Organometallics, 2015, 34, 2550-2557. ChemistrySelect, 2018, 3, 3939-3942.

A. J. Martínez-Martínez, A. R. Kennedy, R. E. Mulvey and C. T. O'Hara, Science, 2014, 834, 834-837.

A. J. Martínez-Martínez, S. Justice, B. J. Fleming, A. R. Kennedy, I. D. H. Oswald and C. T. O'Hara, Sci. Adv., 2017, 3, e1700832.

L. Davin, A. Hernán-Gómez, C. Mclaughlin, A. R. Kennedy, R. McLellan and E. Hevia, Dalton Trans., 2019, 48, 8122 8130.

M. Fairley, L. Davin, A. Hernán-Gómez, J. García-Álvarez, C. T. O'Hara and E. Hevia, Chem. Sci., 2019, 10, 5821-5831. M. De Tullio, A. Hernán-Gómez, Z. Livingstone, W. Clegg, A. R. Kennedy, R. W. Harrington, A. Antiñolo, A. Martínez, F. Carrillo-Hermosilla and E. Hevia, Chem. Eur. J, 2016, 22, 17646-17656.

D. R. Armstrong, W. Clegg, A. Hernán-Gómez, A. R. Kennedy, Z. Livingstone, S. D. Robertson, L. Russo and E. Hevia, Dalton Trans., 2014, 43, 4361-4369.

A. Hernán-Gómez, T. D. Bradley, A. R. Kennedy, Z. Livingstone, S. D. Robertson and E. Hevia, Chem. Commun., 2013, 49, 8659-8661.

J. Jacques, A. Collet and S. H. Wilen, Enantiomers, Racemates and Resolutions, John Wiley \& Sons, New York, 1981.

S. Srisanga and J. H. ter Horst, Cryst. Growth Des., 2010, 10 1808-1812.

C. Li, P. Chen, S. Hsu, C. Lin, H. Huang and B. Ko, J. Organomet. Chem., 2012, 716, 175-181.

P. García-Álvarez, A. R. Kennedy, C. T. O. Hara, K. Reilly and G. M. Robertson, Dalton Trans., 2011, 40, 5332-5341. M. Ángeles Fuentes, A. Zabala, A. R. Kennedy and R. E. Mulvey, Chem. Eur. J., 2016, 22, 14968-14978. V. Snieckus, Chem. Rev., 1990, 90, 879-933. 